\title{
Pronounced dys-autonomic symptoms announcing a primary Sjögren's syndrome
}

\author{
F. Masini ${ }^{1}$, L. Monaco ${ }^{1}$, K. Gjeloshi ${ }^{1}$, E. Pinotti ${ }^{1}$, R. Ferrara ${ }^{1}$, P.C. Pafundi', \\ M. Santini ${ }^{1}$, T. Salvatore ${ }^{1}$, G. Cuomo ${ }^{2}$ \\ ${ }^{1}$ Internal Medicine, Department of Advanced Medical and Surgical Sciences, \\ University of Campania "Luigi Vanvitelli", Napoli, Italy; \\ 2Internal Medicine, Department of Precision Medicine, University of Campania "Luigi Vanvitelli", Napoli, Italy
}

\begin{abstract}
SUMMARY
Sjögren's syndrome (SS) is an autoimmune disease that involves the nervous system in about $20 \%$ of cases. In 25-92\% of patients affected by Sjögren's syndrome, neurological symptoms may precede the sicca syndrome. A 65-year-old male presented with a seven-month history of episodes of near-syncope, constipation, anhidrosis, disabling fatigue and asthenia. Physical examination was unremarkable, whilst the ECG revealed sinus bradycardia. Laboratory tests showed lymphopenia and normal inflammatory markers.

In order to assess a potential autonomic neuropathy, "Deep Breathing Test" (E/I 1.02), "Lying to Standing Test" (R/R' 0.95), and "Orthostatic Hypotension Tests" (T 120s Systolic reduction >20 mmHg and Diastolic reduction $>10 \mathrm{mmHg}$ ) were performed, all of which were abnormal. ECG Holter monitoring revealed sinus bradycardia, and right bundle branch block with 24-h blood pressure monitoring revealing a diurnal hypotensive profile.

The patient reported a three-month history of worsening dry mouth. On physical examination, the patient had anisocoria in response to light stimulation. Auto-antibody testing was performed to evaluate the presence of any autoimmune disease. The results of these studies included an abnormal elevation of ANA (1:320 speckled pattern), Ro/SS-a (>240U/l), and La/SS-b (162 U/ml) antibodies.

The patient was discharged with a diagnosis of "Autonomic Neuropathy Most Likely Due to Primary Sjögren's Syndrome (SS)" and started the immunotherapy. After one month, he reported a significant improvement in his symptoms with a concomitant normalization of his "Orthostatic Hypotension Tests."

This case underlines the potential for dys-autonomic symptoms to precede the onset of sicca syndrome in patients with Sjogren's Syndrome.
\end{abstract}

Key words: Primary Sjögren's syndrome; dys-autonomic symptoms.

Reumatismo, 2020; 72 (2): 111-114

\section{BACKGROUND}

jögren Syndrome (SS) is an autoimmune disease of unknown etiology. SS may involve several organ systems with most notable effects on the exocrine gland function $(1,2)$.

The nervous system is involved in about $20 \%$ of SS cases. In $25-92 \%$ of patients affected by Sjogren's syndrome, neurological symptoms may precede the onset of sicca syndrome (3). Several studies have reported a close relationship between SS and signs of neuropathy (both autonomic and peripheral).

The most common neurological symptoms are represented by neurosensory and motor peripheral neuropathy, sensorial ganglionopathy (sensorial ataxic neuropathy) (4), trigeminal neuropathy, neurosensorial auditory deficit, small fiber neuropathy, transversal myelitis, dysautonomia, anosmia and ageusia. In addition, over half of the patients with Sjogren syndrome have pupil abnormalities (5), orthostatic hypotension (6), hypo/anhidrosis (3).

With particular regard to the involvement of orthostatic hypotension symptoms, a study by NG et al. on 21 patients affected by primary SS (pSS) compared to 21 controls (7) showed a close relationship between autonomic dysfunction and altered
Corresponding author: Giovanna Cuomo Department of Precision Medicine University of Campania "Luigi Vanvitelli", Napoli, Italy

E-mail: giovanna.cuomo@unicampania.it 
blood pressure in pSS patients vs. controls. Given Sjogren's syndrome may manifest in several non-specific ways, the patient may be misdiagnosed. On this basis, we present a clinical case with a rare clinical presentation of this rheumatologic disease. Our patient is a rare case displaying a broad range of dys-autonomic symptoms in the initial manifestation of disease, whilst sicca syndrome, more often suggestive of the disease, appeared later.

\section{CASE REPORT}

A 65-year-old male was seen because of a seven-month history of episodes of nearsyncope, constipation, hypohidrosis, disabling fatigue, and asthenia. His past history revealed hypothyroidism due to Hashimoto's thyroiditis. He was under treatment with levothyroxine. Physical examination was negative, except for dry, dehydrated skin. His vital signs revealed a temperature of $36.5^{\circ} \mathrm{C}$, blood pressure of $90 / 60 \mathrm{mmHg}$, and a respiratory rate of 20 breaths per minute. The ECG revealed sinus bradycardia of 55 beats per minute (bpm) and right bundle-branch block (RBBB). Laboratory findings showed lymphocytopenia (WBC 9008/ $\mu \mathrm{L}$, with $90 \%$ granulocytes and $3 \%$ lymphocytes $),$ CRP $(0.3 \mathrm{mg} / \mathrm{dL}), \mathrm{ESR}(20 \mathrm{~mm} /$ $\mathrm{hr}$ ), while the other inflammatory markers were normal. In order to investigate the presence of any endocrine disorders, several hormone panels were also performed, showing euthyroidism (FT3 $2.0 \mathrm{pg} / \mathrm{mL}$, FT4 $12.6 \mathrm{pg} / \mathrm{mL}$, TSH $1.685 \mu \mathrm{UI} / \mathrm{mL}, \mathrm{Ab}$ anti-thyroglobulin $1630.0 \mathrm{UI} / \mathrm{mL}, \mathrm{Ab}$ antithyreoperoxidase $592.5 \mathrm{UI} / \mathrm{mL}$ ), hypogonadotropic hypogonadism (LH $1.6 \mathrm{mIU} / \mathrm{mL}$, FSH $2.7 \mathrm{mIU} / \mathrm{mL}$, prolactin $15.9 \mathrm{ng} / \mathrm{mL}$, testosterone $133 \mathrm{ng} / \mathrm{dL}$ ), and normal adrenocortical function (serum cortisol 8 am $17.5 \mu \mathrm{g} / \mathrm{dL}$, aldosterone $6.8 \mathrm{pg} / \mathrm{dL}$, ACTH $8.5 \mathrm{pg} / \mathrm{mL}$ ). Imaging examinations, including thyroid gland ultrasonography, revealed mild goiter with coarse echotexture and an overall vascularity increase, whilst brainMRI showed anterior pituitary hypoplasia. In order to assess a potential autonomic neuropathy, a "Deep Breathing Test" (E/I 1.02), "Lying to Standing Test" (R/R'
0.95), and "Orthostatic Hypotension Test" (T 120s, Systolic reduction $>20 \mathrm{mmHg}$, and Diastolic reduction $>10 \mathrm{mmHg}$ ) were performed, all of which were abnormal. ECG Holter monitoring revealed sinus bradycardia, with a mean heart rate of 55 bpm (HR min. $47 \mathrm{bpm}$ at 10.14 a.m. HR max. $70 \mathrm{bpm}$ at 01.54 a.m.) and RBBB. Holter blood pressure 24-hour monitoring showed a diurnal hypotensive profile (mean arterial pressure (MAP) 110/64 $\mathrm{mmHg}$ and HR 49 bpm, diurnal MAP 109/64 mmHg, HR 49 bpm; nocturnal MAP 131/75 mmHg and HR $46 \mathrm{bpm}$ ), whilst the echocardiogram revealed a mild mitral and tricuspid valvular failure. The head-up-tilt test to assess the cardiac sympathetic and adrenergic vasomotor functions showed severe orthostatic hypotension $\left(15^{\text {th }}\right.$ minute PA78/50 $\mathrm{mmHg}$ and HR $55 \mathrm{bpm}$ ). Deficiency of vitamin B12 or thiamine, alcohol dependence along with toxic, and drug-induced autonomic neuropathy were excluded. Based upon clinical history and physical examination, specific tests were ordered to investigate infectious causes of autonomic neuropathy, such as HIV and VDRL/ TPHA, which were negative. Serum amyloid A1 and 24-h proteinuria were within the normal reference ranges.

A paraneoplastic syndrome was also excluded by pan-imaging the patient with a contrast-enhanced, total-body CT scan, esophagogastroduodenoscopy (EGD), colonoscopy, abdomen ultrasound which were normal. Specific tumor markers (Beta2microglobulin, LDH, CA 15-3, CA 19-9, Calcitonin, CEA, Chromogranin A, Gastrin, Monoclonal immunoglobulins, PSA, NSE, TPA, Cyfra 21-1) were assessed, which were also normal. Upon further assessment, the patient reported a threemonth history of worsening dry mouth, whilst on physical examination anisocoria in response to light stimulation was observed as well. Laboratory panels for autoimmune diseases were performed, showing elevated ANA (1:320 speckled pattern), Ro/SS-a (>240U/l) and La/SS-b (162 U/ $\mathrm{ml}$ ) antibodies. Anti-GAChr (anti-ganglionic acetylcholine receptor antibodies) were absent. 
Although symptoms, such as dry eyes, were not reported, the "Schirmer test" revealed insufficient tear production in both eyes (RE $5 \mathrm{~mm}$, LE $3 \mathrm{~mm}$ ). Minor salivar gland biopsy was instead rejected.

Given this particular clinical picture, differential causes of autonomic neuropathy were investigated: metabolic, nutritional, autoimmune, toxic, drug-induced, infectious, genetic, paraproteinemia, paraneoplastic and idiopathic. The patient was discharged with a diagnosis of "Autonomic Neuropathy Most Likely Due to Primary Sjögren's syndrome (SS)" and started a therapy with Prednisone $25 \mathrm{mg} / \mathrm{kg}$ and Hydroxychloroquine $200 \mathrm{mg}$ BID PO. After one month, he reported a significant improvement in symptoms, such as asthenia, fatigue and dry mouth. Also orthostatic hypotension symptoms decreased significantly with normalization of his orthostatic vital signs and no further near-syncopal events. The patient was monitored in follow-up visits scheduled every three months over the past two years. At each follow up visit, several laboratory tests were monitored, among which: blood cell count, electrolytes, liver function, ESR, CRP, C3 and C4 proteins, immunoglobulin levels, LDH, beta2 microglobulin, urine analysis, and tumor markers. Additionally, electrocardiogram, autonomic neuropathy testing, abdomen ultrasound and chest CT were performed after two years and were normal. Follow-up is still ongoing.

\section{DISCUSSION AND CONCLUSIONS}

Although several reports described neurological symptoms in SS patients, it has been widely assumed that autonomic impairment in SS is typically mild, or even subclinical, if recognized at all.

From a pathologic point of view, we can observe a poor correlation between the degree of destruction of the exocrine glands and their function. Moreover, the reduced exocrine secretion might be potentially due to the interference of neuronal signaling $(8,9)$. In particular, the etiopathogenesis of dysautonomia is characterized by inflammatory cytokines interfering with the cholinergic neurotransmission as well as with the destruction of the autonomic nervous fibers of the glandular cells (10). An additional sign is also the presence of an inflammatory infiltrate of $\mathrm{T}$ lymphocytes in the ganglion and the nervous root of the autonomic nervous system (11).

In our clinical case, further investigations to complete the diagnostic process and exclude paraneoplastic autonomic neuropathy would have been appropriate. However, they are not available in all hospitals (e.g., nicotinic acetylcholine ganglionic antibodies, anti-Neuronal Nuclear Antibodies [ANNA-1 or anti-Hu], quantitative pseudomotor axon reflex testing, Valsalva maneuver). In particular, paraneoplastic autonomic neuropathy, resulting from the autoimmune destruction of autonomic postganglionic neurons, may precede the diagnosis of a malignant neoplasm and occur as a component of paraneoplastic neuropathy with anti-Hu antibodies in $23 \%$ of patients (12). Given this evidence, it is recommended to perform full screening tests for SS or other rheumatologic diseases in every patient with symptoms consistent with autonomic neuropathy, whenever the initial assessment does not provide any clear evidence. Currently, there are no clinical recommendations that suggest this consideration. We propose to include laboratory tests in the screening, such as anti-nuclear antibodies, anti-SSA and anti-SSB. Should the laboratory findings be unrevealing, further assessments to be considered should include more specific tests, such as salivary gland biopsy, Schirmer's test, and Rose Bengal $(3,12)$.

Symptoms related both to SS and the autonomic dysfunction in SS are immune-responsive to corticosteroids and hydroxychloroquine (13), as also shown in our patient, who has already responded favorably to this treatment after one month.

Moreover, in the current literature, clear clinical improvements in symptoms of dysautonomia were observed after repeated immunotherapy with intravenous immunoglobulin (IVIG), sometimes alongside Rituximab (13).

In conclusion, our case underlines the clinical importance of dysautonomic symptoms, 
before the onset of sicca syndrome, and highlights the favorable response to immune-modulating therapy in the treatment of the autonomic dysfunction arising from SS.

\section{Conflict of interest}

The authors have no conflicts of interest to disclose.

\section{REFERENCES}

1. Mavragani CP, Moutsopoulos HM. Sjögren's syndrome. Annu Rev Pathol. 2014; 9: 273-85.

2. Voigt A, Sukumaran S, Nguyen CQ. Beyond the glands: an in-depth perspective of neurological manifestations in Sjögren's syndrome. Rheumatology (Sunnyvale). 2014; 2014: pii: S4-010.

3. Berkowitz AL, Samuels MA. The neurology of Sjögren's syndrome and the rheumatology of peripheral neuropathy and myelitis. Pract Neurol. 2014; 14: 14-22.

4. Pereira PR, Viala K, Maisonobe $\mathrm{T}$, et al. Sjögren sensory neuronopathy (Sjögren ganglionopathy): long-term outcome and treatment response in a series of 13 cases. Medicine (Baltimore). 2016; 95: e3632.

5. Bhagwan S, Bhagwan B, Moodley A. Bilateral tonic pupils as the initial manifestation of Sjögren's syndrome. Neuroophthalmology. 2015; 39: 248-52.
6. Kovács L, Paprika D, Tákacs R, et al. Cardiovascular autonomic dysfunction in primary Sjögren's syndrome. Rheumatology (Oxford). 2004; 43: 95-9.

7. Ng WF, Stangroom AJ, Davidson A, et al. Primary Sjögrens syndrome is associated with impaired autonomic response to orthostasis and sympathetic failure. QJM 2012; 105: 1191-9.

8. Humphreys-Beher MG, Brayer J, Yamachika $\mathrm{S}$, et al. An alternative perspective to the immune response in autoimmune exocrinopathy: induction of functional quiescence rather than destructive autoaggression. Scand J Immunol. 1999; 49: 7-10.

9. Dawson LJ, Fox PC, Smith PM. Sjögrens syndrome-the non-apoptotic model of glandular hypofunction. Rheumatology (Oxford). 2006; 45: 792-8.

10. Waterman SA, Gordon TP, Rischmueller M. Inhibitory effects of muscarinic receptor autoantibodies on parasympathetic neurotransmission in Sjögren's syndrome. Arthritis Rheum. 2000; 43: 1647-54.

11. Fox RI, Stern M. Sjögren's syndrome: mechanisms of pathogenesis involve interaction of immune and neurosecretory systems. Scand J Rheumatol Suppl. 2002; 116: 3-13.

12. Sumida T, Azuma N, Moriyama M, et al. Clinical practice guideline for Sjögren's syndrome 2017. Mod Rheumatol. 2018; 28: 383-408.

13. Goodman BP. Immunoresponsive autonomic neuropathy in Sjögren syndrome-case series and literature review. Am J Ther. 2019; 26: e66-e71. 